\title{
Comparative Analysis of the WISC between Two ADHD Subgroups
}

\author{
Yoichiro Kubo ${ }^{1,2}$, Tetsufumi Kanazawa ${ }^{1,3,4} \bowtie$, Yasuo Kawabata' ${ }^{1}$ Koyuki Tanaka1, Tatsushi Okayama1, \\ Miho Fujta ${ }^{1}$, Atsushi Tsutsumi ${ }^{1}$, Shingo Yokota ${ }^{2}$, and Hiroshi Yoneda ${ }^{1}$ \\ ${ }^{1}$ Department of Neuropsychiatry, Osaka Medical College, Osaka, Japan \\ ${ }^{2}$ Department of Psychiatry, Han-nan Hospital, Osaka, Japan \\ ${ }^{3}$ Department of Psychiatry, The University of Melbourne, Melbourne, Australia \\ ${ }^{4}$ Florey Institute, Oxidation Biology Unit, Melbourne, Australia
}

Objective The prevalence of attention deficit/hyperactivity disorder (ADHD) in school-age children is 7.2\%, and ADHD is divided into clinical subtypes.

Methods The current study explored whether specific cognitive profiles as assessed using the Wechsler Intelligence Scale for Children (WISC)-IV could be obtained for each clinical ADHD subtype (ADHD-Inattentive type and ADHD-Combined type) and investigated the correlation between WISC scores and parental age at their children's birth or birthweight. The enrolled sample comprised 12 ADHD-I and 15 ADHD-C subjects.

Results An impaired Processing Speed Index was found in ADHD-I. The age of the father at the child's birth and birthweight positively correlated with the full scale intelligence quotient (FSIQ) score in the WISC assessment.

Conclusion Inattentiveness within the behaviors of the children with ADHD-I is partly due to the impaired processing speed, therefore effective support for ADHD will be conducted if educator decreases their speaking speed. Since biological basis of ADHD is still largely unknown, future studies using both psychological and biological methods will reveal the etiology of ADHD. These scientific assessments will provide information for more effective approaches in the care of children with ADHD.

Psychiatry Investig 2018;15(2):172-177

Key Words Neurodevelopmental disorder, Child and adolescence psychiatry, Cognitive sciences.

\section{INTRODUCTION}

Attention deficit/hyperactivity disorder (ADHD) is a common psychiatric disorder in school-age children, with a prevalence of 7.2\%. ${ }^{1}$ However, symptom presentation is not limited to school ages. Based on a national survey in the US, ${ }^{2} 4.4 \%$ of the adult population satisfies the DSM-IV ADHD diagnostic criterion. However, the risk of overdiagnosing ADHD or excessive medication has recently become a substantial issue. ${ }^{3}$ Historically, the formulation of the concept of ADHD

Received: April 24, 2017 Revised: June 5, 2017

Accepted: July 12, 2017 Available online: November 21, 2017

$\triangle$ Correspondence: Tetsufumi Kanazawa, MD, $\mathrm{PhD}$

Department of Psychiatry, Osaka Medical College, 2-7 Daigaku-machi, Takatsuki, Osaka 569-8686, Japan

Tel: +81-72-683-1221, Fax: +81-72-683-4810, E-mail: psy052@osaka-med.ac.jp

(c) This is an Open Access article distributed under the terms of the Creative Commons Attribution Non-Commercial License (http://creativecommons.org/licenses/bync/4.0) which permits unrestricted non-commercial use, distribution, and reproduction in any medium, provided the original work is properly cited. began with a case report by Sir GF Still in $1902 .{ }^{4}$ Still reported 43 cases with "defect of moral control associated with general impairment of intellect," which represents the hyperactive or impulsive behavioral feature of ADHD in childhood. In 1980, attention deficit disorder (ADD) was included in the DSMIII. ADD subtypes (ADD/H with hyperactivity or ADD/WO without hyperactivity) should be added to the criteria for $\mathrm{ADD}$ diagnosis. In the revised text of the DSM-III (DSM-IIIR), the first description of $\mathrm{ADHD}$ was added, and the recently updated criterion, the DSM-5, has inherited the concept of ADHD. Due to the historical process, the subtypes, such as ADHD$\mathrm{H}$ (primarily hyperactive/impulsive), ADHD-I (primarily inattentive), or ADHD-C (combined), should be identified at ADHD diagnosis. For example, several studies have found more severe and internalized symptoms in children with $\mathrm{AD}$ HD-C than those in children with ADHD- $\mathrm{I}^{5,6}$ because children with ADHD-I have only impaired attention, whereas children with ADHD-C have impairments in both hyperactivity-im- 
pulsivity and attention. ${ }^{7}$ Additionally, practical care is different among the subtypes. As detecting ADHD subtypes is crucial for planning medication, prognostic prediction and improving quality of life, $\mathrm{ADHD}$ must be evaluated not only on the basis of symptoms but also with psychological assessments, including cognitive batteries.

In the current retrospective survey, we aimed to identify different features between two ADHD subtypes (ADHD-C and ADHD-I) using the Wechsler Intelligence Scale for Children (WISC) assessment. The WISC is typically administered to children (aged 5 years and older) and adolescents (less than 17 seventeen years old) to evaluate their full scale intelligence quotient (FSIQ), which represents children's general intellectual ability and cognitive functions. The WISC assessment consists of four factors: the Processing Speed Index (PSI), the Verbal Comprehension Index (VCI), the Perceptual Reasoning Index (PRI), and the Working Memory Index (WMI) (Figure 1).

Another aim was to reveal the relationship between parental age at the children's birth and the subtypes or the subscale scores on the WISC. Recent reports have suggested a higher incidence rate of ADHD (13.1-fold) if the father is more than

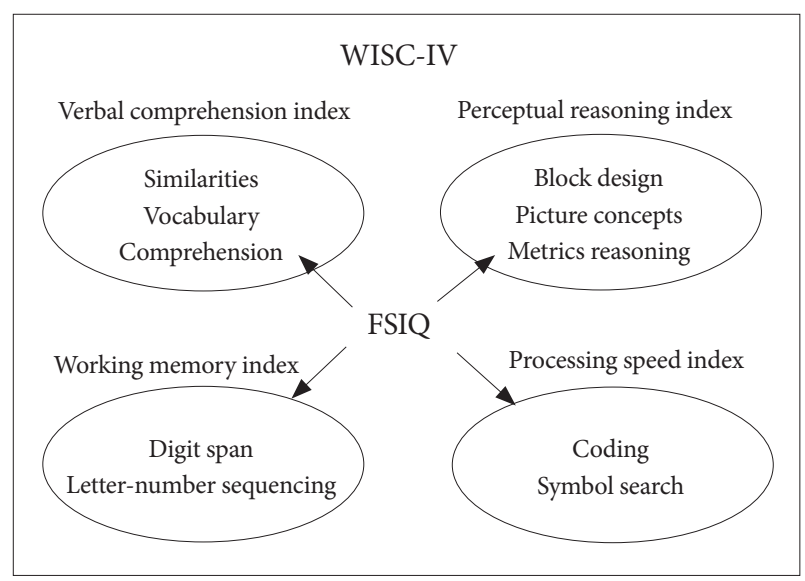

Figure 1. The components of the four WISC-IV subscales. WISC: Wechsler Intelligence Scale for Children, FSIQ: full scale intelligence quotient.
45 years old at the child's birth. ${ }^{8}$ Similarly, compared with fathers aged 25-29 years old, fathers less than 20 years old have an increased risk of having children with ADHD (1.5-fold). ${ }^{9}$ These inconsistent reports motivated us to determine the association between parental age at ADHD children's birth and cognitive features in ADHD. In addition, we evaluated the relationship between birthweight and WISC scores, including the FSIQ and four subclassifications.

\section{METHODS}

During 2014, children meeting the DSM-IV ADHD criteria who visited Han-nan Hospital were analyzed. Han-nan Hospital is located in the west of Japan and has a pediatric psychiatric unit (maximum capacity: 25 children). Each day, an average of 15.1 outpatients visit the pediatric psychiatry hospital, and 1.1 children become new patients.

ADHD patients with a FSIQ (assessed by WISC) less than 70 were omitted from further analysis. Twenty-seven patients (22 boys and 5 girls; average age of 9.7 years) were selected for clinical record collection (Table 1). This retrospective analysis was approved by the Ethical Committee at Han-nan Hospital

Table 1. WISC assessment of the participants $(\mathrm{N}=27)$

\begin{tabular}{lcl}
\hline & Average score & SD \\
\hline FSIQ & 96.2 & \pm 15.3 \\
Verbal comprehension index & 96.3 & \pm 12.6 \\
Perceptual reasoning index & 100.2 & \pm 19.1 \\
Block design & 9.7 & \pm 3.8 \\
Picture concepts & 10.2 & \pm 2.8 \\
Metrics reasoning & 10.3 & \pm 3.6 \\
Working memory index & 94.2 & \pm 14.1 \\
Digit span & 8.7 & \pm 2.4 \\
Letter-number sequencing & 9.1 & \pm 3.6 \\
Processing speed index & 94.7 & \pm 11.6 \\
\hline
\end{tabular}

FSIQ: full scale intelligence quotient, SD: standard deviation

Table 2. Group comparison of WISC assessment $(\mathrm{N}=27)$

\begin{tabular}{|c|c|c|c|c|c|}
\hline \multirow{2}{*}{ WISC-IV } & ADHD-C & \multirow{2}{*}{$\mathrm{SD}$} & ADHD-I & \multirow{2}{*}{$\mathrm{SD}$} & \multirow{2}{*}{$\mathrm{t}$} \\
\hline & Average score $(\mathrm{N}=15)$ & & Average score $(\mathrm{N}=12)$ & & \\
\hline FSIQ & 99.1 & \pm 18.3 & 92.6 & \pm 10.2 & -1.1 \\
\hline Verbal comprehension index & 95.3 & \pm 14.3 & 97.6 & \pm 10.6 & 0.5 \\
\hline Perceptual reasoning index & 104.9 & \pm 22.6 & 94.3 & \pm 11.9 & -1.5 \\
\hline Working memory index & 95.7 & \pm 15.2 & 92.3 & \pm 13.0 & -0.6 \\
\hline Processing speed index & 99.3 & \pm 11.1 & 89.0 & \pm 9.7 & $-2.5^{*}$ \\
\hline Coding & 10.1 & \pm 2.7 & 7.8 & \pm 2.4 & $-2.2^{*}$ \\
\hline Symbol search & 10.0 & \pm 3.4 & 8.3 & \pm 1.9 & -1.5 \\
\hline
\end{tabular}

${ }^{*} \mathrm{p}<0.05$. WISC: Wechsler Intelligence Scale for Children, ADHD-C: attention deficit/hyperactive disorder (combined type), ADHD-I: attention deficit/hyperactive disorder (inattentive type), FSIQ: full scale intelligence quotient, SD: standard deviation 
(H27-04).

Statistical procedures included correlation analysis of the relationship between WISC results and parental age or birthweight. Unpaired t-test was used when the two assigned groups (ADHD-I and ADHD-C) were compared in terms of the results from the WISC subscales. Data were analyzed using JMP Pro ${ }^{\circledR}$ statistical software.

\section{RESULTS}

Twenty-seven patients were assigned as ADHD-I $(\mathrm{n}=12)$ or ADHD-C ( $\mathrm{n}=15)$ based on the DSM-IV criterion. The average parental age at birth was 31.2 years for fathers and 28.6 years for mothers ( $n=26$, respectively). Unpaired t-tests revealed significant differences between the two groups (ADHD-I and ADHD-C) on the PSI $(t=-0.25, \mathrm{p}<0.05)$ but not FSIQ. On the subscale analysis, a significant difference was found only for the Coding scale subtype $(\mathrm{t}=-2.2, \mathrm{p}<0.05)$ (Table 2).

The correlation analysis comparing parental age at birth

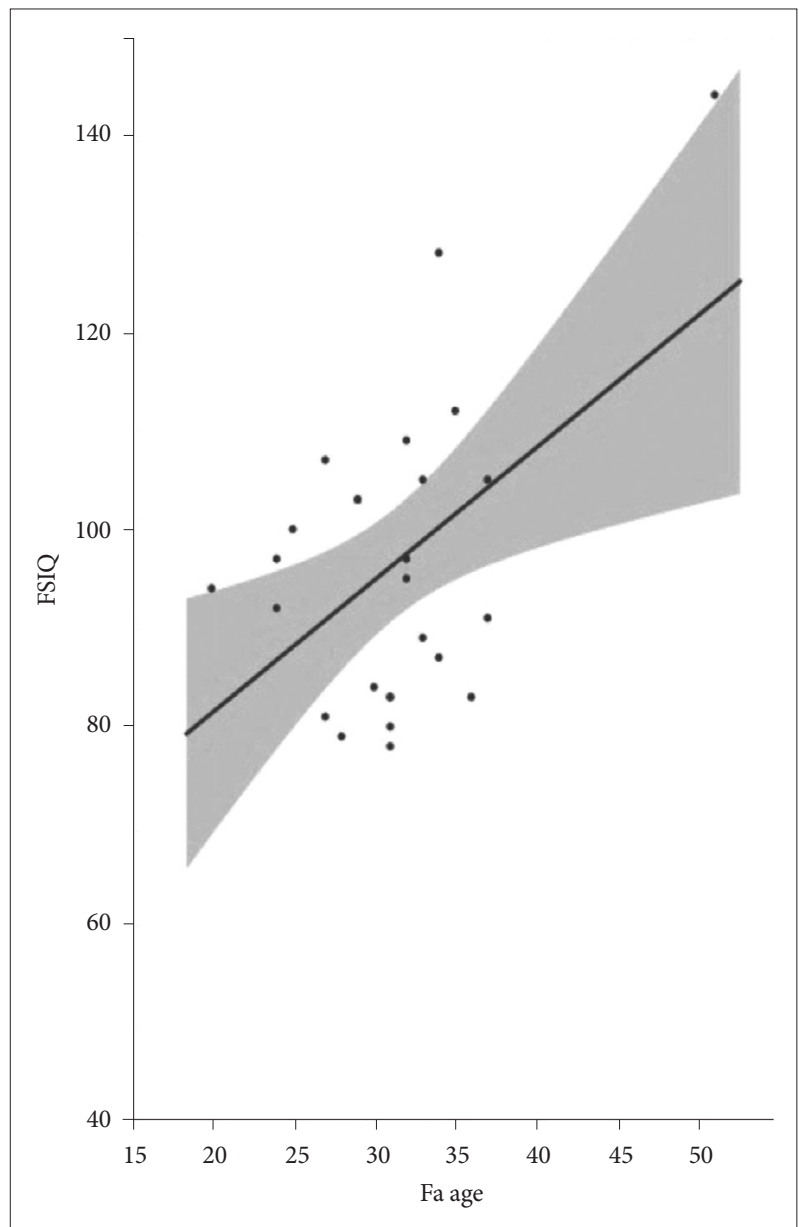

Figure 2. Scatterplot of FSIQ and father's age at ADHD child's birth $(\mathrm{N}=27)$. $\mathrm{FSIQ}$ : full scale intelligence quotient, $A D H D$ : attention deficit/hyperactive disorder. and birthweight with the FSIQ and WISC revealed that FSIQ and father's age at birth were significantly correlated $(r=0.50$, $\mathrm{p}=0.0091$ ) (Figure 2). The subscale analysis indicated that both the PRI $(\mathrm{r}=0.46, \mathrm{p}=0.0174)$ and WMI $(\mathrm{r}=0.49, \mathrm{p}=0.0116)$ of the WISC correlated with father's age at the child's birth. In addition, the birthweight of the 27 patients was significantly correlated with the FSIQ ( $\mathrm{r}=0.40, \mathrm{p}=0.0364)$ (Figure 3 ) and WMI $(\mathrm{p}=0.0149)$ within the subscales. Moreover, if birthweight was analyzed only, including full-term births $(\mathrm{n}=20$, birth between 37 weeks and 41 weeks), the FSIQ, PRI ( $\mathrm{p}<0.01)$, VCI, WMI, and PSI $(\mathrm{p}<0.05)$ were correlated (Table 3$)$.

\section{DISCUSSION}

The purpose of the current retrospective survey was to eval-

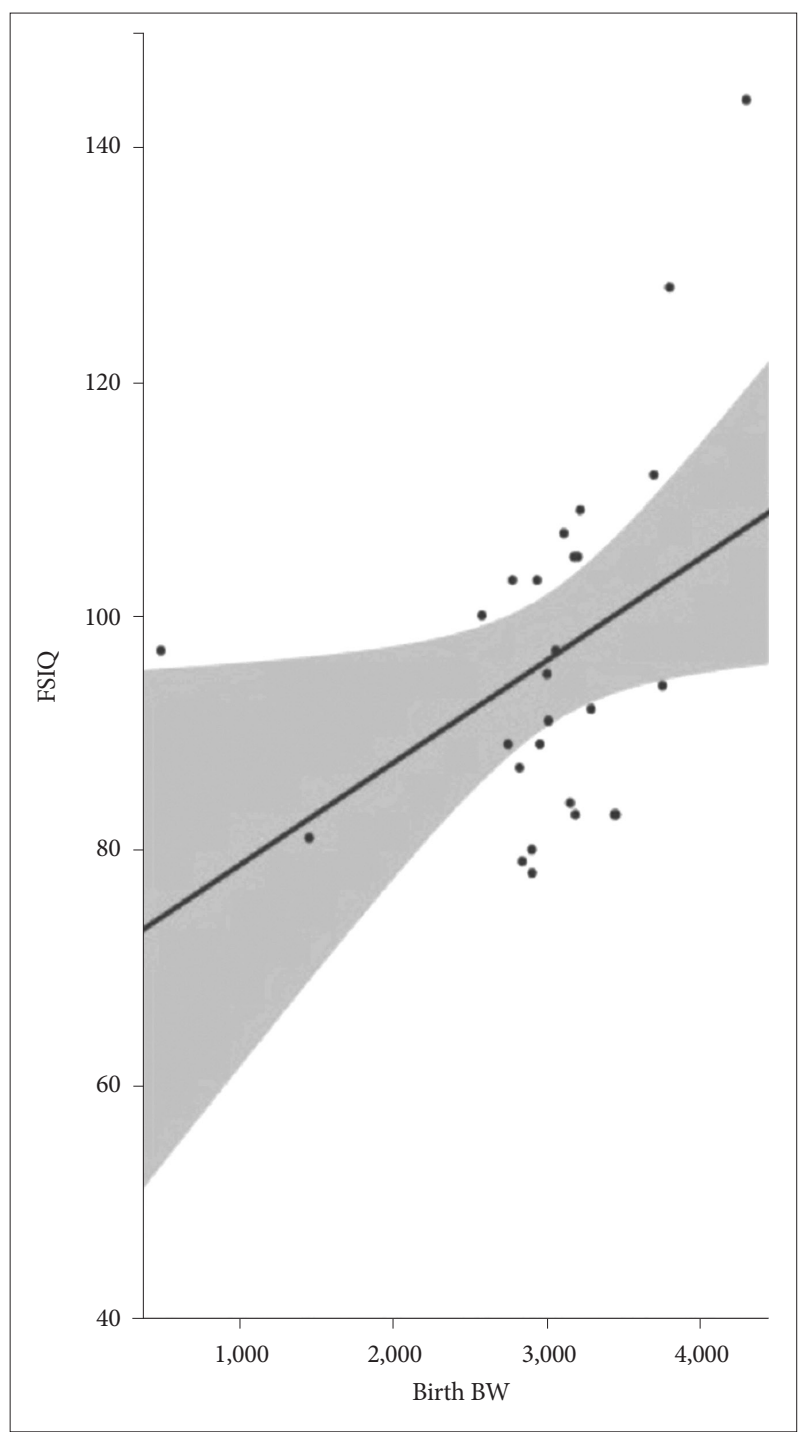

Figure 3. Scatterplot of FSIQ and bodyweight at ADHD child's birth $(\mathrm{N}=27)$. FSIQ: full scale intelligence quotient, $A D H D$ : attention deficit/hyperactive disorder. 
Table 3. WISC assessment and Parental age and birthweight

\begin{tabular}{|c|c|c|c|c|c|c|}
\hline Subscales in WISC test & $\begin{array}{l}\text { Average score } \\
\quad(\mathrm{N}=27)\end{array}$ & SD & $\begin{array}{c}\text { Father's age } \\
\text { at child's birth }\end{array}$ & $\begin{array}{l}\text { Mother's age } \\
\text { at child's birth }\end{array}$ & $\begin{array}{l}\text { Birthweight } \\
(\mathrm{N}=27)\end{array}$ & $\begin{array}{l}\text { Birthweight (full- } \\
\text { term birth } \mathrm{N}=20 \text { ) }\end{array}$ \\
\hline FSIQ & 96.2 & \pm 15.3 & $\mathrm{r}=0.50^{* *}$ & $\mathrm{r}=0.15$ & $\mathrm{r}=0.40^{*}$ & $\mathrm{r}=0.67^{* *}$ \\
\hline Verbal comprehension index & 96.3 & \pm 12.6 & $\mathrm{r}=0.32$ & $\mathrm{r}=0.24$ & $\mathrm{r}=0.22$ & $\mathrm{r}=0.52^{*}$ \\
\hline Perceptual reasoning index & 100.2 & \pm 19.1 & $\mathrm{r}=0.46^{*}$ & $\mathrm{r}=0.05$ & $\mathrm{r}=0.34$ & $\mathrm{r}=0.60^{* *}$ \\
\hline Block design & 9.7 & \pm 3.8 & $\mathrm{r}=0.45^{*}$ & & & \\
\hline Picture concepts & 10.2 & \pm 2.8 & $\mathrm{r}=0.35$ & & & \\
\hline Metrics reasoning & 10.3 & \pm 3.6 & $\mathrm{r}=0.38$ & & & \\
\hline Working memory index & 94.2 & \pm 14.1 & $\mathrm{r}=0.49^{*}$ & $\mathrm{r}=0.33$ & $\mathrm{r}=0.46^{*}$ & $\mathrm{r}=0.56^{*}$ \\
\hline Digit span & 8.7 & \pm 2.4 & $\mathrm{r}=0.44^{*}$ & & $\mathrm{r}=0.45^{*}$ & \\
\hline Letter-number sequencing & 9.1 & \pm 3.6 & $\mathrm{r}=0.29$ & & $\mathrm{r}=0.28$ & \\
\hline Processing speed index & 94.7 & \pm 11.6 & $\mathrm{r}=0.34$ & $\mathrm{r}=-0.21$ & $\mathrm{r}=0.32$ & $\mathrm{r}=0.46^{*}$ \\
\hline
\end{tabular}

${ }^{*} \mathrm{p}<0.05,{ }^{* *} \mathrm{p}<0.01$. WISC: Wechsler Intelligence Scale for Children, FSIQ: full scale intelligence quotient, SD: standard deviation

uate WISC scores among children with ADHD. Determining levels of intelligence in children with ADHD-I and ADHD-C will provide information for more appropriate involvement in their care. Treatment strategies are slightly different among ADHD subtypes. For instance, moderate to high doses of methylphenidate (Ritalin) are used for children with ADHD$\mathrm{C}$, whereas low doses are helpful for most children with ADHD-I. ${ }^{10}$

The results indicate similar FSIQ scores between the two subtypes on the WISC, whereas PSI scores were significantly higher in ADHD-C than those in ADHD-I. Processing speed represents how quickly a person can perform simple or automatic cognitive tasks; thus, children with hyperactive behavior showed faster speeds on the cognitive tasks. The higher PSI scores in ADHD-C are consistent with a previous study. ${ }^{11}$ Thaler et al. ${ }^{12}$ reported that WISC profiles in children with ADHD fit five cluster models in hierarchical cluster analysis. Within the clusters, two clusters (named Reduced PSI and Below Average WMI/PSI) indicated that low WISC-IV PSI was associated with ADHD-I. In other words, children with a lower PSI were recognized as inattentive. A similar trend in the PRI was found, although the difference was not significant. A higher PRI in ADHD-C indicates better outcomes with an educational approach that includes visualization or modeling, especially for children with ADHD-C. By contrast, the VCI is the only subscale in which the scores of ADHD-I exceed those of ADHDC. This finding potentially suggests that ADHD-I affects verbal and auditory communication.

ADHD-associated inattentiveness is believed to be due to a deficit in the locus coeruleus-norepinephrine system (LC-NE system $^{13}$ ) because differences in P3 (synonymous name: P300, Event Related Potential) amplitudes has been frequently found in people with inattentiveness. ${ }^{14-16}$ However, concluding that inattentiveness in ADHD is directly related to altered P3 am- plitudes is difficult because deficits in P3 amplitudes are observed not only in ADHD-I patients but also in boys with disinhibitory disorders, such as conduct disorder, antisocial personality disorder, alcoholism, or nicotine dependence. ${ }^{17}$ The locus coeruleus (ceruleus) is located in the pons and is involved in physiological responses to stress or damage. Through the release of norepinephrine, the LC-NE system regulates many brain functions involved in attention, memory, emotions, and behavioral flexibility. ${ }^{18,19}$ Similar findings have also been obtained with electroencephalogram (EEG) measurements. Previous research has shown that alpha wave patterns in individuals with ADHD are different from those in agematched controls. ${ }^{20}$ Furthermore, increased theta and slower alpha waves, which are associated with inefficient brain processing or disturbed awareness, have been repeatedly found in subjects with ADHD; however, this feature is not unique to ADHD. ${ }^{21}$ People with obsessive compulsive disorders, learning disorders, or some types of dementia tend to show similar EEG patterns. Inattention is one of the domains regulated by the LC-NE system; therefore, the specific etiology of inattentiveness in ADHD patients should be studied more intensively with an electrophysiological approach.

Additional studies using functional neuroimaging approaches, such as SPECT, PET and fMRI, have repeatedly detected associations between ADHD and abnormalities in the fronto-striatal circuitry, including the dorsolateral prefrontal cortex (DLPFC), ventrolateral prefrontal cortex (VLPFC), dorsal anterior cingulate cortex (dACC), and striatum. ${ }^{21}$ Although the apparent deficit in fronto-striatal circuitry may be downstream of the initially impaired brain regions, ADHD pathology is at least related to this circuitry. Beyond the fronto-striatal model, newer approaches with Diffusion Tensor Image (DTI) of the default mode network have shown deficits in many other brain regions, such as visual networks. For in- 
stance, a 33-year follow-up study of ADHD children detected decreased cortical thickness in the medial occipital cortex. ${ }^{22}$ Although abnormalities in the medial occipital cortex (BA18, BA19) have been repeatedly shown in earlier neuroimaging studies, ${ }^{23,24}$ sufficient attention has not been given to this region. High-resolution MRI has indicated reduced gray matter volume in the bilateral occipital cortex ${ }^{25}$ in people with $\operatorname{ADHD}(n=31)$ compared with healthy controls $(n=31)$; therefore, the reduced PSI could be partly explained by impaired visual function in individuals with ADHD.

Inattentiveness in ADHD-I is characterized by a "sluggish cognitive tempo" (SCT). ${ }^{26-28}$ SCT items on the DSM-IV Field Trial for ADHD were discarded due to insufficient predictive power to distinguish ADHD subtypes ${ }^{29}$; however, SCT has been re-evaluated because SCT is consistent with the features of sluggishness, drowsiness, spaciness, lethargy, and hypoactivity in children with ADHD-I. ${ }^{10}$ Additionally, SCT refers to frequently observed conditions such as daydreaming, having difficulty staying awake in certain situations, and becoming easily bored, easily confused or more lethargic than others. A recent systematic review of $\mathrm{SCT}^{30}$ has reported that SCT affects an individual's cognitive functioning (e.g., social interactions or academic achievement), but the lack of standards in SCT measurements across studies was also revealed. Moreover, SCT has a hypothesized link to impaired processing speed. Most studies ${ }^{5,26,31,32}$ have suggested a link between SCT and slow processing speed; however, some researchers have questioned whether neuropsychological/behavioral assessments of cognitive tempo have been applied. ${ }^{33}$ The lowed processing speed in ADHD-I found in our study is based on WISC performance. Thus, further assessment using an appropriate SCT measurement is warranted in the future.

Regarding the parental ages at birth of the children with ADHD, self-reported father's ages at the child's birth and the FSIQ from the sample were significantly associated $(r=0.50$, $\mathrm{p}=0.0091$ ), whereas no significant correlation with mother's age was found. Recent epidemiological studies in Sweden (2.6 million people, ${ }^{8}$ and Denmark (3 million people) ${ }^{9}$ have found higher incidence rates of schizophrenia and autism with older fathers ( $>45$ years old). However, the incidence of ADHD is slightly different from the incidence of these two disorders. Offspring born to men over the age of 45 years were 5\% and $43 \%$ less likely to have a diagnosis of ADHD in the Danish and Swedish studies, respectively. ${ }^{34}$ As a reference group was not included in the current study, our findings cannot be treated as a replication of these two large-scale epidemiological studies. However, the FSIQ in the ADHD sample $(\mathrm{n}=27)$ was associated with the father's age at the child's birth. In general, an inverted U-shaped relationship is observed between paternal age and IQ of offspring. ${ }^{35} \mathrm{~A}$ recent genomic analysis has found that de novo mutations carry a large effect on higher rates of autism spectrum disorder, which is regarded as one of the reasons why older paternal age increases the risk of schizophrenia or autism spectrum disorders in offspring. Future work including both genomic and psychological assessments of ADHD children will reveal the biological basis of the altered cognitive functions in ADHD in detail.

Several limitations should be addressed. Most importantly, a limited number of ADHD subjects were enrolled in this study. This limitation is due to the brief duration of the sample recruitment (1 year) of patients with first visits during 2014. Therefore, future studies should expand the recruitment duration to include more psychological analysis data. Another limitation is the lack of data from ADHD-H (primarily hyperactive/impulsive). Most previous studies have focused on the difference between ADHD-C and ADHD-I because cases with pure $\mathrm{ADHD}-\mathrm{H}$ phenotypes are rarely seen in school-age children. ${ }^{1}$

Children with ADHD-C adapt more easily to education with visualized methods (e.g., graphs, figures, and behavioral modeling); children with ADHD-I have a harder time adapting because of their limited PRI. Similar results will be obtained if educators choose verbal communication, although educators should decrease their speaking speed because we found worse PSI in children with ADHD-I on the current WISC assessment. A recent meta-analysis aimed at accurately estimating the prevalence of ADHD concluded that the prevalence is $7.2 \%$ and $6.6-7.8 \%$ at $95 \% \mathrm{CI}^{1}{ }^{1} \mathrm{ADHD}$ prevalence varies among schools, cultures, and countries. Children with ADHD have trouble concentrating or sitting still in classrooms. Therefore, effective involvement in the care of children with ADHD is needed, and the current psychological assessment findings could be helpful for developing support for these children.

\section{REFERENCES}

1. Thomas R, Sanders S, Doust J, Beller E, Glasziou P. Prevalence of attention-deficit/hyperactivity disorder: a systematic review and meta-analysis. Pediatrics 2015;135:e994-e1001.

2. Kessler RC, Adler L, Barkley R, Biederman J, Conners CK, Demler O, et al. The prevalence and correlates of adult ADHD in the United States: results from the National Comorbidity Survey Replication. Am J Psychiatry 2006;163:716-723.

3. Bonati M, Reale L. Reducing overdiagnosis and disease mongering in ADHD in Lombardy. BMJ 2013;347:f7474.

4. Still GF. Some abnormal psychical conditions in children: the Goulstonian lectures. Lancet 1902;12:58.

5. Barkley RA, DuPaul GJ, McMurray MB. Comprehensive evaluation of attention deficit disorder with and without hyperactivity as defined by research criteria. J Consult Clin Psychol 1990;58:775-789.

6. Gross-Tsur V, Goldzweig G, Landau YE, Berger I, Shmueli D, Shalev RS. The impact of sex and subtypes on cognitive and psychosocial aspects of ADHD. Dev Med Child Neurol 2006;48:901-905.

7. Solanto MV, Gilbert SN, Raj A, Zhu J, Pope-Boyd S, Stepak B, et al. Neurocognitive functioning in $\mathrm{AD} / \mathrm{HD}$, predominantly inattentive 
and combined subtypes. J Abnorm Child Psychol 2007;35:729-744.

8. D’Onofrio BM, Rickert ME, Frans E, Kuja-Halkola R, Almqvist C, Sjolander A, et al. Paternal age at childbearing and offspring psychiatric and academic morbidity. JAMA Psychiatry 2014;71:432-438.

9. McGrath JJ, Petersen L, Agerbo E, Mors O, Mortensen PB, Pedersen CB. A comprehensive assessment of parental age and psychiatric disorders. JAMA Psychiatry 2014;71:301-309.

10. Diamond A. Attention-deficit disorder (attention-deficit/ hyperactivity disorder without hyperactivity): a neurobiologically and behaviorally distinct disorder from attention-deficit/hyperactivity disorder (with hyperactivity). Dev Psychopathol 2005;17:807-825.

11. Fenollar-Cortés J, Navarro-Soria I, González-Gómez C, García-Sevilla J. Cognitive Profile for Children with ADHD by Using WISC-IV: Subtype Differences. Rev Psicodidáctica 2015;20:157-176.

12. Thaler NS, Bello DT, Etcoff LM. WISC-IV profiles are associated with differences in symptomatology and outcome in children with ADHD. J Atten Disord 2013;17:291-301.

13. Sroubek A, Kelly M, Li X. Inattentiveness in attention-deficit/hyperactivity disorder. Neurosci Bull 2013;29:103-110.

14. Holcomb PJ, Ackerman PT, Dykman RA. Auditory event-related potentials in attention and reading disabled boys. Int J Psychophysiol 1986;3:263-273.

15. Howells FM, Stein DJ, Russell VA. Synergistic tonic and phasic activity of the locus coeruleus norepinephrine (LC-NE) arousal system is required for optimal attentional performance. Metab Brain Dis 2012;27: 267-274.

16. Kratz O, Studer P, Malcherek S, Erbe K, Moll GH, Heinrich H. Attentional processes in children with ADHD: an event-related potential study using the attention network test. Int J Psychophysiol 2011;81:82-90.

17. Iacono WG, Carlson SR, Malone SM, McGue M. P3 event-related potential amplitude and the risk for disinhibitory disorders in adolescent boys. Arch Gen Psychiatry 2002;59:750-757.

18. Malenka RC, Nestler EJ, Hyman, SE, Sydor A, Brown RY. Molecular Neuropharmacology: A Foundation for Clinical Neuroscience. NY: McGraw-Hill Medical; 2009.

19. Benarroch EE. The locus ceruleus norepinephrine system: functional organization and potential clinical significance. Neurology 2009;73: 1699-1704.

20. Nazari MA, Wallois F, Aarabi A, Berquin P. Dynamic changes in quantitative electroencephalogram during continuous performance test in children with attention-deficit/hyperactivity disorder. Int J Psychophysiol 2011;81:230-236.

21. Bush G, Valera EM, Seidman LJ. Functional neuroimaging of attention-deficit/hyperactivity disorder: a review and suggested future directions. Biol Psychiatry 2005;57:1273-1284.

22. Proal E, Reiss PT, Klein RG, Mannuzza S, Gotimer K, Ramos-Olaza- gasti MA, et al. Brain gray matter deficits at 33-year follow-up in adults with attention-deficit/hyperactivity disorder established in childhood. Arch Gen Psychiatry 2011;68:1122-1134.

23. Dickstein SG, Bannon K, Castellanos FX, Milham MP. The neural correlates of attention deficit hyperactivity disorder: an ALE meta-analysis. J Child Psychol Psychiatry 2006;47:1051-1062.

24. Vance A, Silk TJ, Casey M, Rinehart NJ, Bradshaw JL, Bellgrove MA, et al. Right parietal dysfunction in children with attention deficit hyperactivity disorder, combined type: a functional MRI study. Mol Psychiatry 2007;12:826-832, 793.

25. Ahrendts J, Rüsch N, Wilke M, Philipsen A, Eickhoff SB, Glauche V, et al. Visual cortex abnormalities in adults with ADHD: a structural MRI study. World J Biol Psychiatry 2011;12:260-270.

26. Carlson CL, Mann M. Sluggish cognitive tempo predicts a different pattern of impairment in the attention deficit hyperactivity disorder, predominantly inattentive type. J Clin Child Adolesc Psychol 2002;31:123129.

27. Carlson CL, Mann M. Attention-deficit/hyperactivity disorder, predominantly inattentive subtype. Child Adolesc Psychiatr Clin N Am 2000; 9:499-510, vi.

28. McBurnett K, Pfiffner LJ, Frick PJ. Symptom properties as a function of ADHD type: an argument for continued study of sluggish cognitive tempo. J Abnorm Child Psychol 2001;29:207-213.

29. Frick PJ, Lahey BB, Applegate B, Kerdyck L, Ollendick T, Hynd GW, et al. DSM-IV field trials for the disruptive behavior disorders: symptom utility estimates. J Am Acad Child Adolesc Psychiatry 1994;33:529-539.

30. Mueller AK, Tucha L, Koerts J, Groen Y, Lange KW, Tucha O. Sluggish cognitive tempo and its neurocognitive, social and emotive correlates: a systematic review of the current literature. J Mol Psychiatry 2014;2:5.

31. Lahey BB, Schaughency EA, Hynd GW, Carlson CL, Nieves N. Attention deficit disorder with and without hyperactivity: comparison of behavioral characteristics of clinic-referred children. J Am Acad Child Adolesc Psychiatry 1987;26:718-723.

32. Desman C, Petermann F, Hampel P. Deficit in response inhibition in children with attention deficit/hyperactivity disorder (ADHD): impact of motivation? Child Neuropsychol 2008;14:483-503.

33. Bauermeister JJ, Barkley RA, Bauermeister JA, Martínez JV, McBurnett $\mathrm{K}$. Validity of the sluggish cognitive tempo, inattention, and hyperactivity symptom dimensions: neuropsychological and psychosocial correlates. J Abnorm Child Psychol 2012;40:683-697.

34. D’Onofrio BM, Lichtenstein P. The Age Gauge: Older Fathers Having Children. In Cerebrum: the Dana forum on brain science. Dana Foundation, 2014, July.

35. Malaspina D, Reichenberg A, Weiser M, Fennig S, Davidson M, Harlap $S$, et al. Paternal age and intelligence: implications for age-related genomic changes in male germ cells. Psychiatr Genet 2005;15:117-125. 